\title{
Lung cancer screening by single-shot dual-energy subtraction using flat-panel detector
}

\author{
Hiroshi Mogami ${ }^{1}\left[\right.$ Yumiko Onoike $^{1} \cdot$ Hiroshi Miyano $^{1} \cdot$ Kenji Arakawa $^{1} \cdot$ Hiromi Inoue $^{1} \cdot$ Kouji Sakae $^{1} \cdot$ \\ Toshiaki Kawakami ${ }^{1}$
}

Received: 14 January 2021 / Accepted: 20 June 2021 / Published online: 26 June 2021

(c) The Author(s) 2021

\begin{abstract}
Purpose The purpose of this study was to evaluate the usefulness of single-shot dual-energy subtraction (DES) method using a flat-panel detector for lung cancer screening

Materials and methods The subjects were 13,315 residents (5801 males and 7514 females) aged 50 years or older (5097 years, with an intermediate value of 68 years) who underwent lung cancer screening for a period of 1 year and 6 months from January 2019 to June 2020. We investigated whether the number of lung cancers detected, the detection rate, and the rate of required scrutiny changed, when DES images were added to the judgment based on conventional chest radiography. Results When DES images were added, the number and percentage of cancer detection increased from $16(0.12 \%)$ to 23 $(0.17 \%)(P<0.05)$. Five of the newly detected 7 lung cancers were in the early stages of resectable cancer. The rate of participants requiring scrutiny increased slightly from 1.1 to $1.3 \%$.

Conclusion DES method improved the detection of lung cancer in screening. The increase in the percentage of participants requiring scrutiny was negligible.
\end{abstract}

Keywords Lung cancer $\cdot$ Chest radiograph $\cdot$ Energy subtraction $\cdot$ Lung cancer screening $\cdot$ Flat-panel detector

\section{Abbreviations \\ CT Computed tomography \\ FPD Flat-panel detector \\ GGA Ground-glass attenuation \\ DQE Detective quantum efficiency}

\section{Introduction}

The effectiveness of lung cancer screening by chest radiography is considered to be limited [1], and some researches on lung cancer screening by low-dose CT are ongoing [2, 3]. The detection of lung cancer by chest radiography is not sufficient and the detection rate has been reported to be $70-80 \%[4,5]$. This is mostly due to the fact that the ribs and clavicles overlap the lungs on chest radiography, making it difficult to detect nodular shadows [6]. Recently, several methods have been developed to remove bones from

Hiroshi Mogami

mogamih@eghca.or.jp

1 Ehime General Healthcare Association, 1-10-5, Misake-cho, Matsuyama, Ehime 790-0814, Japan chest radiography. One is software-based bone suppression (BS) method [7]. The other is DES method, which removes bone by acquiring two images with different energies and subtracting them [8-10]. Of these, one is a single-exposure method using storage phosphor system and the other is a double-exposure method using an FPD. The former has problems with image quality and processing performance [11], while the latter is not widely used due to problems such as increased radiation dose and motion artifacts caused by double-exposure [10, 12]. In the present study, we performed lung cancer screening using a newly developed system that can generate DES images by a single-exposure method with two overlapping FPDs and verified the effectiveness of the system.

\section{Materials}

This study was conducted on residents who underwent lung cancer screening for a period of 1 year and 6 months from January 2019 to June 2020. Chest X-rays were taken on site using a health screening bus. A total of 13,315 residents (5801 males and 7514 females) aged 50 years and older 
(50-97 years, intermediate age 68 years) with or without smoking were included in the study.

\section{Methods}

The generator was Radnext CS (Hitachi Medico, Tokyo, Japan). The X-ray receiving system was CALNEO Dual (Fujifilm, Tokyo, Japan). The tube voltage was $130 \mathrm{kVp}$, the tube current was $80 \mathrm{~mA}$, and automatic imaging system was performed using a photo timer. Based on the phantom experiment, the timer was extended by about $20 \%$ longer than usual to ensure the signal-to-noise ratio and graininess of DES images. The surface dose without DES was $0.210 \mathrm{mGy}$ at $130 \mathrm{kV}, 2.4 \mathrm{mAs}$, while with DES it was $0.252 \mathrm{mGy}$ at $130 \mathrm{kV}, 2.8 \mathrm{mAs}$. These were measured with the application software, Sdec v6. The National diagnostic reference levels in Japan (2020) are $0.30 \mathrm{mGy}$ for frontal chest radiography, so both values are within that range. The panel size was $46 \times 46 \mathrm{~cm}$, and the spatial resolution was 5 pixels $/ \mathrm{mm}$, corresponding to $2300 \times 2300$ pixels per image. The distance between the tube and the subject was $200 \mathrm{~cm}$. The FPD places CsI in the foreground to produce conventional chest images and GOS in the background to produce high-energy images. The high-energy image was emphasized, weighted and subtracted to produce a soft tissue image and a bone image. After exposure, it took about $7 \mathrm{~s}$ for DES images to be generated, and DES images and conventional images were displayed on the monitor in turn for image confirmation. There was no burden on the radiology technician, and the participants did not have to wait. For reading, we used a viewer with two $5 \mathrm{M}$ monitors (ScrEagle, Miura, Hiroshima, Japan) for observation and inputting judgments: one for the current image and the other for the past image.
The reading experiment was conducted by a single reader. For double reading, another readers read the chest radiography without DES images. This decision was not used in this experiment. First, only the conventional image was read and judged. Next, on a different day (3 days or less), the conventional image was read with DES images added and judged blindly. DES images consisted of soft tissue images with the bone removed and bone images with the soft tissue removed. Conventional images, soft tissue images, and bone images were read in this order. In the observation of soft tissue images, there was no obvious criterion for determining that the lesion required scrutiny, but if a region of interest was found, the current and past images were reviewed carefully to determine if it was a new lesion or not. If any changes were observed over time, the lesion required scrutiny. Findings of merely faint increased attenuation or less than $5 \mathrm{~mm}$ did not require scrutiny which were considered to be of little clinical significance. Abnormalities in the ribs and calcification of the lungs and pleura can be confused with lung cancer nodules by conventional imaging alone, but with DES method, they disappear on soft tissue images and appear on bone images, so we were able to confidently determine that there was no need for scrutiny (Fig. 1).

We examined whether the presence or absence of DES images changed the percentage of participants who required scrutiny and the number of cancers detected.

\section{Results}

There were 121 participants who required scrutiny in the absence of DES images and remained unchanged with the addition of DES images. There were 51 participants who did not require scrutiny in the absence of DES images but
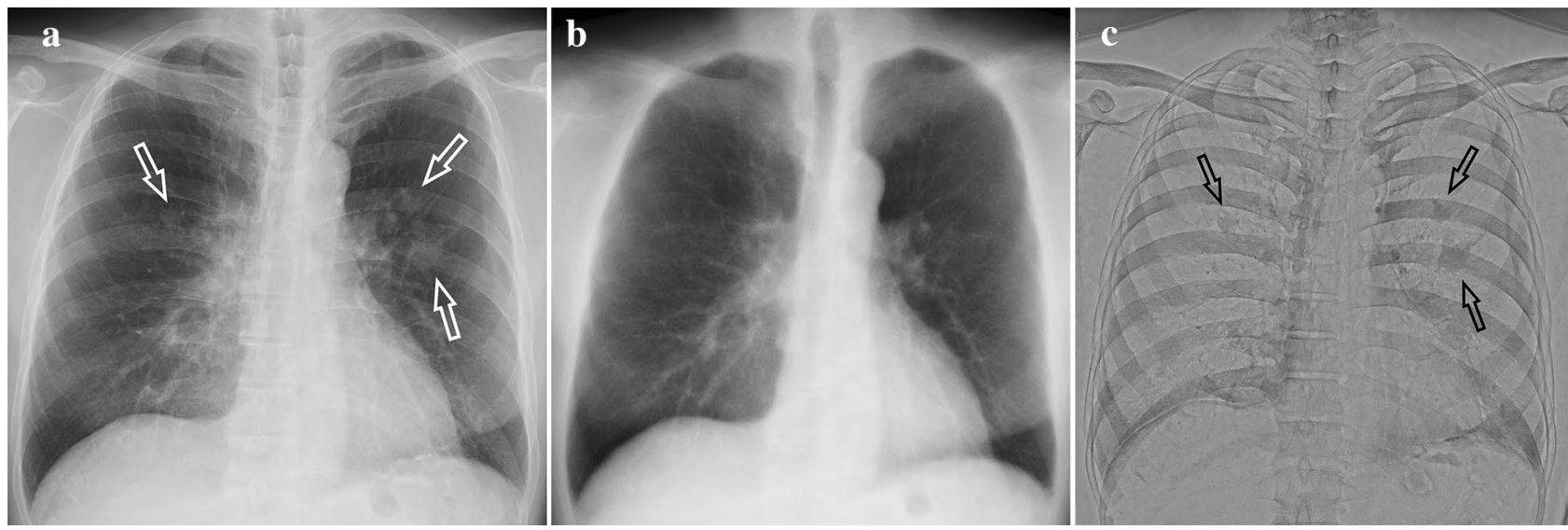

Fig. 1 Male, $80 \mathrm{~s}$, pleural calcifications. a Conventional radiography showed bilateral nodular shadows (arrows). b The soft tissue image showed no nodular shadows. $\mathbf{c}$ The bone image showed bilateral nodular shadows(arrows), which were determined to be pleural calcifications 
Table 1 Change in number of the participants requiring scrutiny with and without DES images

\begin{tabular}{llll}
\hline & DES $-/+$ & DES $-/+$ & DES $-/+$ \\
\hline Scrutiny & pos $\rightarrow$ pos & neg $\rightarrow$ pos & pos $\rightarrow$ neg \\
Number & 121 & 51 & 23
\end{tabular}

$D E S$ - without DES images, DES + with DES images, pos positive, neg negative

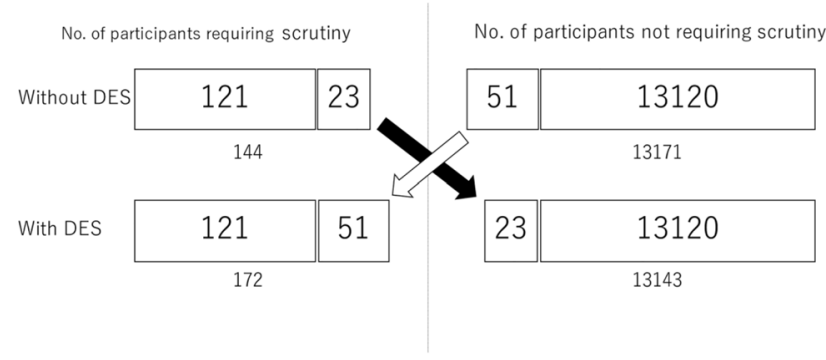

Fig. 2 Change in number of the participants requiring scrutiny with and without DES images

Table 2 Final determination of scrutiny with and without DES images

\begin{tabular}{lll}
\hline & Percentage (\%) & Number \\
\hline DES - & 1.1 & 144 \\
DES + & 1.3 & 172 \\
\hline
\end{tabular}

$D E S$ - without DES images, DES + with DES images

Table 3 Percentage and number of newly detected lung cancers

\begin{tabular}{lll}
\hline & Percentage $(\%)$ & Number \\
\hline DES - & 0.12 & 16 \\
DES + & 0.17 & 23 \\
\hline
\end{tabular}

$D E S$ - without DES images, DES + with DES images

required it with the addition of DES images. There were 23 participants who required scrutiny in the absence of DES images but no longer required it with the addition of DES images (Table 1 and Fig. 2).

Finally, the percentage of participants requiring scrutiny increased slightly from 1.1 to $1.3 \%$ (Table 2). The number of cancers detected was 16 without DES images, but 7 new cancers were detected using DES images, bringing the total to 23 . The cancer detection rate increased from 0.12 to $0.17 \%$ (Table 3 ). There was a statistically significant difference between the two groups by McNemar's test $(P<0.05)$. Based on these results, the positive predictive value increased from 11 (16/144) to 13\% (23/172) (n.s.).
Table 4 Newly detected lung cancers with DES images

\begin{tabular}{lllll}
\hline Age/gender & Histology & Size $(\mathrm{mm})$ & Stage & Therapy \\
\hline $60 \mathrm{~s} / \mathrm{M}$ & Adenoca & 30 & IB & Ope \\
$80 \mathrm{~s} / \mathrm{F}$ & Adenoca & 30 & IA1 & Ope \\
$80 \mathrm{~s} / \mathrm{M}$ & Adenoca & 30 & IA3 & Ope \\
$60 \mathrm{~s} / \mathrm{F}$ & Adenoca & 22 & IA2 & Ope \\
$80 \mathrm{~s} / \mathrm{M}$ & Unknown & 13 & IA1 & RT \\
$70 \mathrm{~s} / \mathrm{M}$ & Sq & 10 & IVA post F/U & CT \\
$50 \mathrm{~s} / \mathrm{F}$ & Mts & 10 & & CT \\
\hline
\end{tabular}

Adenoca adenocarcinoma, $S q$ squamous cell carcinoma, $F / U$ follow up, Ope operation, $R T$ radiation therapy, $C T$ chemotherapy, $M t s$ metastasis

There were five stage I patients, four of whom had adenocarcinoma and underwent resection, and one patient underwent radiotherapy without histological proof. Another patient had squamous cell carcinoma and was followed up for 4 months without histological proof, and was treated with chemotherapy because of tumor growth and metastasis. The other patient had lung metastasis from breast cancer. (Table 4 and Figs. 3, 4 and 5).

\section{Discussion}

Recently, methods to remove bone from chest radiography has been developed, and several reading experiments have been reported to investigate the detection rate of lung nodules [10-13]. This is the first report of the use of DES method for screening of lung cancer. With the addition of DES images, new lung cancers were detected. Most of the newly detected lung cancers were resectable early-stage cancers. With the addition of DES images, the detection rate of lung cancer has improved by $30 \%$, from 0.11 to $0.17 \%$. However, it was not higher than that of CT. On the other hand, among the lung cancers detected by CT, there were not a few cases of adenocarcinoma in situ and minimally invasive adenocarcinoma, mainly GGA, and the problem of overdiagnosis has been proposed [14]. In the present study, no pure GGA lesions were found. Even with the bone removed, it was not easy to pick up the slight attenuation differences in the GGA lesions on the DES images, which depicted thick, overlapping lung structures on a single 2D image. However, in previous reports $[15,16]$, adenocarcinoma of part-solid type on CT was a useful target for DES method, and the newly discovered cancer in this study included such lesions. In other words, DES images has the potential to detect lifethreatening lung cancer at a time when it is resectable, and if used for lung cancer screening, it is expected to reduce the number of lung cancer deaths. 

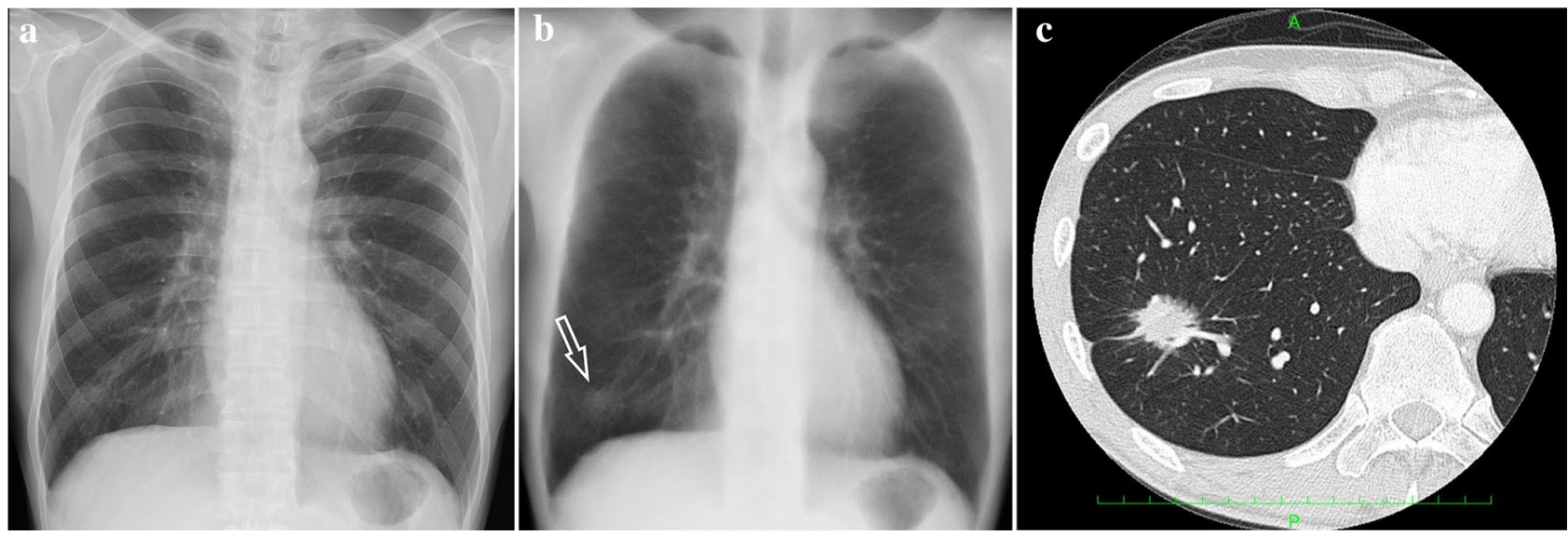

Fig. 3 Male, $60 \mathrm{~s}$, adenocarcinoma, $30 \mathrm{~mm}$. a No abnormal findings were observed on conventional radiography. b The soft tissue image showed a nodular shadow in the right lower lung field (arrow). c CT showed a solid nodular shadow with GGA in the right lower lobe
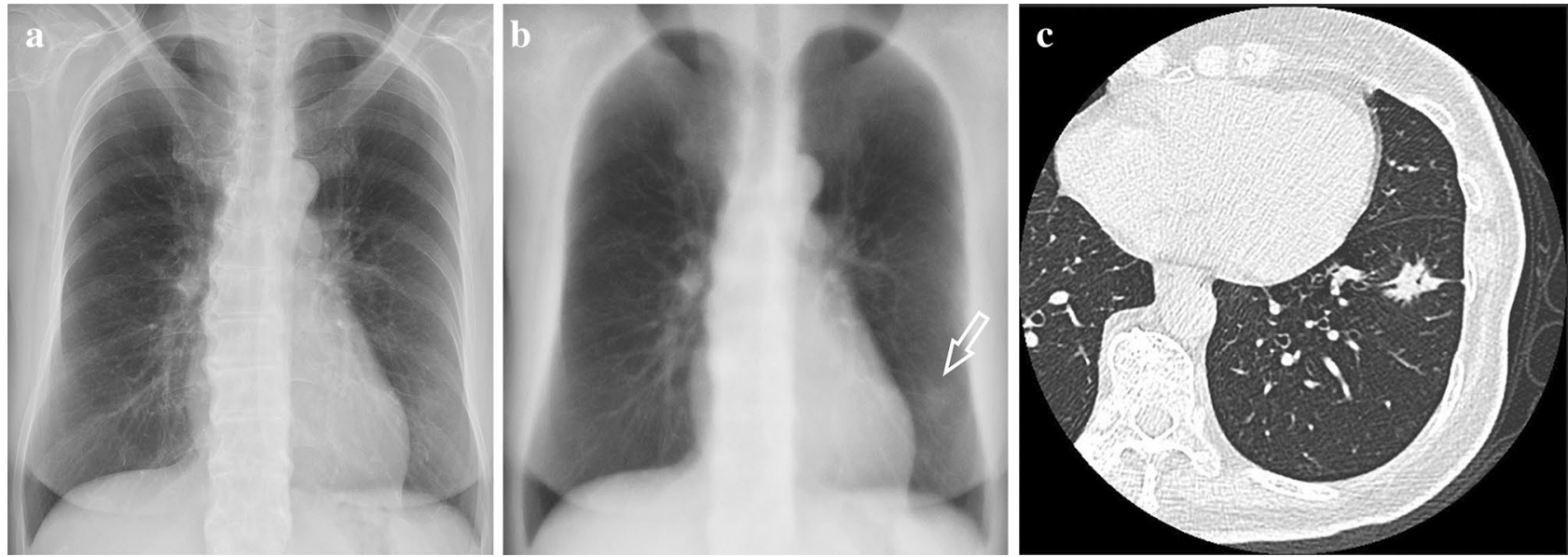

Fig. 4 Female, $60 \mathrm{~s}$, adenocarcinoma, $22 \mathrm{~mm}$. a No abnormal findings were observed on conventional radiography. $\mathbf{b}$ The soft tissue image showed a nodular shadow in the left lower lung field (arrow). c CT showed a nodular shadow in the left lower lobe
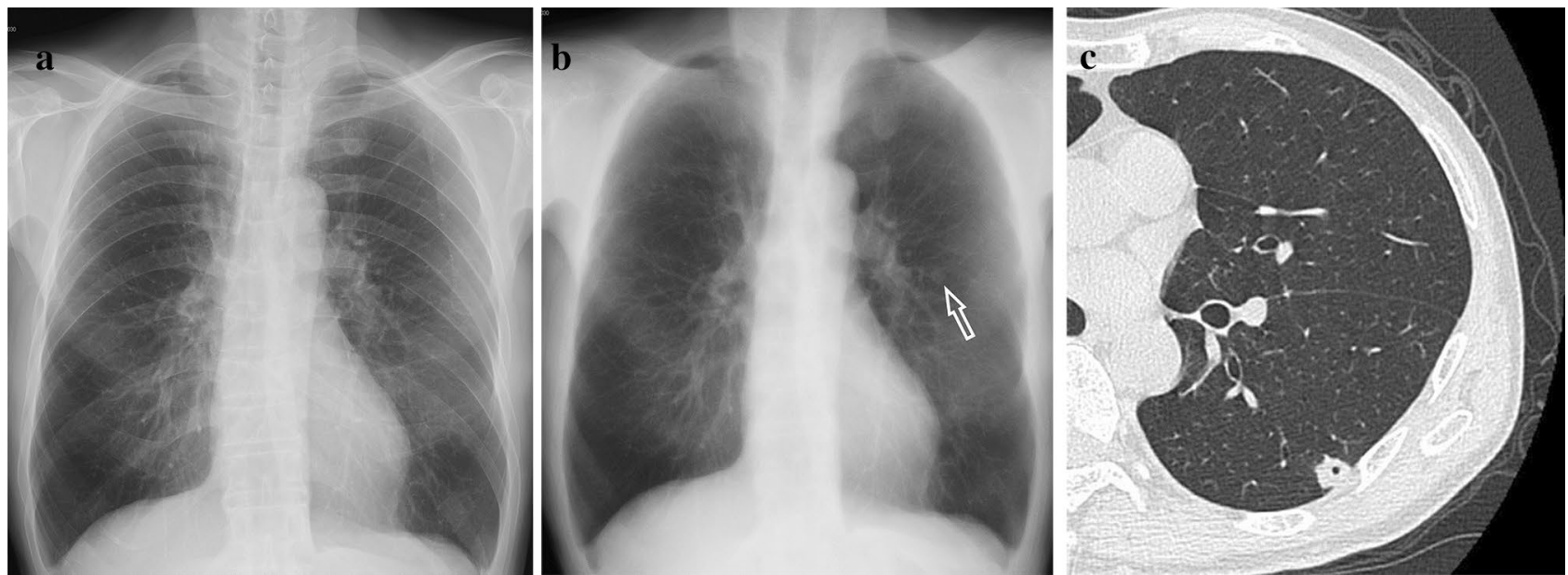

Fig. 5 Male, $70 \mathrm{~s}$, squamous cell carcinoma, $10 \mathrm{~mm}$. a No abnormal findings were observed on conventional radiography. b The soft tissue image showed a nodular shadow in the left middle lung field (arrow). c CT showed a nodular shadow in the left lower lobe 
In soft tissue images, the removal of the ribs made the contours and attenuation differences of the lung cancer nodules much clearer. The X-ray receiving system used in this study was a FPD which has a higher DQE than the system using the storage phosphor system $[17,18]$. In addition, advances in image processing have improved the signal-to-noise ratio over the entire lung field. The radiation dose was only about $20 \%$ higher than that of modern FPDs because of the singleshot nature of the system. The dose is markedly lower than that of the film-screen method, the storage phosphor system or double-shot energy-subtraction system.

The rate of participants requiring scrutiny increased only slightly from 1.1 to $1.3 \%$. The number of participants requiring scrutiny increased by 51 with the addition of soft tissue images. However, this was offset by a decrease of 23 participants due to the observation of bone images. Unlike BS method, which attempts to remove bone by software image processing, DES images are generated from two different energy images and are material discriminating. Therefore, a lesion that disappears in soft tissue images and shows up in bone images is unambiguously a bone or calcified lesion and is excluded from cancer candidacy. This is the advantage over BS method, and it is expected to reduce the rate of requiring scrutiny as much as possible in the screening.

A limitation of this study is that there was only one reader in this experiment, whereas the 2-month pilot study used multiple readers. Since there was a large difference in the judgments of the readers, it was decided to use one reader in this experiment. In this paper, we were able to show the effectiveness of DES method in actual clinical practice under limited conditions, but we were not able to sufficiently verify its universality. We are now waiting for a multi-center validation.

\section{Conclusion}

DES method improved the detection of lung cancer in screening. The increase in the percentage of participants requiring scrutiny was negligible. DES method also minimized the increase in radiation exposure.

Acknowledgements This study was supported by grants from Fuji Film Corporation, Tokyo, Japan.

\section{Declarations}

Ethical approval The ethics committee of our institution approved this study, and informed consent was obtained from all individual participants included in the study.

Open Access This article is licensed under a Creative Commons Attribution 4.0 International License, which permits use, sharing, adaptation, distribution and reproduction in any medium or format, as long as you give appropriate credit to the original author(s) and the source, provide a link to the Creative Commons licence, and indicate if changes were made. The images or other third party material in this article are included in the article's Creative Commons licence, unless indicated otherwise in a credit line to the material. If material is not included in the article's Creative Commons licence and your intended use is not permitted by statutory regulation or exceeds the permitted use, you will need to obtain permission directly from the copyright holder. To view a copy of this licence, visit http://creativecommons.org/licenses/by/4.0/.

\section{References}

1. Oken MM, Hocking WG, Kvale PA, Andriole GL, Buys SS, Churchet TR, et al. Screening by chest radiograph and lung cancer mortality: the prostate, lung, colorectal, and ovarian (PLCO) randmized trial. JAMA. 2011;306(17):1865-73.

2. The National Lung Screening Trial Research Team. Reduced lung-cancer mortality with low-dose computed tomographic screening. N Engl J Med. 2011;365(5):395-409.

3. Harry JK, Carlijn MA, Pim AJ, Scholten ET, Nackaerts K, Heuvelmans MA, et al. Reduced lung-cancer mortality with volume CT screening in a randomized trial. N Engl J Med. 2020;382:503-13.

4. Quekel LG, Kessels AG, Goei R, van Engelshoven JM. Miss rate of lung cancer on the chest radiograph in clinical practice. Chest. 1999;115(3):720-4.

5. Austin JH, Romney BM, Goldsmith LS. Missed bronchogenic carcinoma: radiographic findings in 27 patients with a potentially resectable lesion evident in retrospect. Radiology. 1992;182(1):115-22.

6. Shah PK, Austin JH, White CS, Patel P, Haramati LB, Pearson GD. Missed non-small cell lung cancer: radiographic findings of potentially resectable lesions evident only in retrospect. Radiology. 2003;226:235-41.

7. Li F, Engelmann R, Pesce LL, Doi K, Metz CE, MacMahon H. Small lung cancers: improved detection by use of bone suppression imaging - comparison with dual-energy subtraction chest radiography. Radiology. 2011;261:937-49.

8. Kido S, Nakamura H, Ito W, Shimura K, Kato H. Computerized detection of pulmonary nodules by single-exposure dual energy computed radiography of the chest (part 1). Eur J Radiol. 2002;44(3):198-204.

9. Kido S, Kuriyama K, Kuroda C, Nakamura H, Ito W, Shimura $\mathrm{K}$, et al. Detection of simulated pulmonary nodules by singleexposure dual-energy computed radiography of the chest: effect of a computer-aided diagnosis system (part 2). Eur J Radiol. 2002;44(3):205-9.

10. Uemura M, Miyagawa M, Yasuhara Y, Murakami T, Ikura H, Sakamoto K, et al. Clinical evaluation of pulmonary nodules with dual-exposure dual-energy subtraction chest radiography. Radiat Med. 2005;23(6):391-7.

11. Niklason LT, Hickey NM, Chakraborty DP, Sabbagh EA, Yester MV, Fraser RG, Barnes GT. Simulated pulmonary nodules: detection with dual-energy digital versus conventional radiography. Radiology. 1986;160:589-93.

12. Manji F, Wang J, Norman G, Wang Z, Koff D. Comparison of dual energy subtraction chest radiography and traditional chest $\mathrm{X}$-rays in the detection of pulmonary nodules. Quant Imaging Med Surg. 2016;6(1):1-5.

13 Farkas ZS, Schick A, Cullmann J, Ebner L, Megyeri B, Vock $\mathrm{P}$, et al. Comparison of dual-energy subtraction and electronic bone suppression combined with computer-aided detection on chest radiographs: effect on human observers' performance in nodule detection. Am J Roentgenol. 2013;200:1006-13. 
14. Edward F, Patz J, Paul P, Constantine G. Overdiagnosis in lowdose computed tomography screening for lung cancer. JAMA Intern Med. 2014;174(2):269-74.

15. Ide K, Mogami H, Murakami T, Yasuhara Y, Miyagawa M, Mochizuki H. Detection of lung cancer using single-exposure dual-energy subtraction chest radiography. Radiat Med. 2007;25(5):195-201.

16. Oda S, Awai K, Funama Y, Utsunomiya D, Yanaga Y, Kawanaka $\mathrm{K}$, et al. Effects of dual-energy subtraction chest radiography on detection of small pulmonary nodules with varying attenuation: receiver operating characteristic analysis using a phantom study. Radiat Med. 2010;28:214-9.

17. Garmer M, Hennings SP, Jagar HJ, Schrick F, Loo T, Jacobs A, et al. Digital radiography versus conventional radiography in chest imaging:diagnostic performance of a large-area silicon flat-panel detector in a clinical CT-controlled study. Am J Roentgenol. 2000;174:75-80.

18. Floyd CE, Warp RJ, Dobbins JT 3rd, Chotas HG, Baydush AH, Vargas-Voacec R, et al. Imaging characteristics of amorphous silicon flat-panel detector for digital chest radiography. Radiology. 2001;218:683-8.

Publisher's Note Springer Nature remains neutral with regard to jurisdictional claims in published maps and institutional affiliations. 